\title{
Prognostic Values of Different Clinicopathological Factors and Predictive Models for Penile Carcinoma
}

\author{
Yanxiang Shao* \\ Thongher Lia* \\ Yaohui Wang $\mathbb{D}^{*}$ \\ Kan Wu \\ Xu Hu (D) \\ Yang Liu \\ Shuyang Feng \\ Shangqing Ren \\ Zhen Yang \\ Sanchao Xiong \\ Weixiao Yang \\ Qiang Wei \\ Hao Zeng \\ Xiang Li (D)
}

'Department of Urology, Institute of Urology, West China Hospital, Sichuan University, Chengdu, 61004I, People's Republic of China; ${ }^{2}$ Robotic Minimally Invasive Surgery Center, Sichuan

Academy of Medical Sciences \& Sichuan Provincial People's Hospital, Chengdu, 610072, People's Republic of China; ${ }^{3}$ Department of Urology, Chengdu Second People's Hospital, Chengdu, 61002I, People's Republic of China

*These authors contributed equally to this work
Correspondence: Xiang Li

Department of Urology, Institute of Urology, West China Hospital, Sichuan University, 37 GuoXueXiang, Chengdu, 61004I, People's Republic of China Email xiangli87@hotmail.com
Objective: To evaluate the prognostic factors of penile cancer and the utility of prognostic models.

Methods: We analyzed postoperatively collected data of 311 patients diagnosed with penile cancer. Survival analysis (Kaplan-Meier and cox regression methods) was performed on this cohort. The c-index was used to determine the predictive accuracies of potential prognostic factors. The accuracies of four prognostic models were also evaluated, which were AJCC prognostic stage group for three recent editions, and four nomograms constructed by the Surveillance, Epidemiology, and End Results program (SEER). Two novel nomograms using our data were created and AUC of 2-year survival were determined to compare existing and newly established models.

Results: Tumor site, $\mathrm{T}$ and $\mathrm{N}$ stages, nuclear grade and lymph vascular invasion (LVI) significantly influenced prognosis. The 8th $\mathrm{T}$ and $\mathrm{N}$ stages had better c-indexes than former editions, while no improvement was seen in the 8thAJCC stage group. 6th AJCC+grade nomogram had a higher c-index than other three nomograms (SEER+grade, 6th TNM+grade, and 6th $\mathrm{T}_{1-3} \mathrm{~N}_{0-3}$ +grade nomograms; c-index: 0.831 vs $0.738,0.792$ and 0.781 ). New nomogram 1 included the 8 th $\mathrm{T}$ and $\mathrm{N}$ stages, tumor site, nuclear grade, and LVI, with a c-index of 0.870 . Novel nomogram 2 replaced the T and N stages with the AJCC stage group, which had a lower c-index of 0.855 . The order of prediction accuracy of 2-year survival in the old and new models is consistent with the c-index results.

Conclusion: Tumor site, stages, grade, and LVI play important roles in predicting survival of penile cancer. The 8th stages have better predictive accuracy than former editions. We proposed two models with better predictive accuracy than former models; specifically, nomogram 1 may be a more precise and convenient tool for predicting penile cancer outcomes.

Keywords: penile cancer, TNM stage, survival, nomogram, external validation

\section{Introduction}

Penile squamous cell carcinoma (PSCC) is a relative rare disease in USA and Europe, with the overall incidence is $<1$ in 100,$000 ;{ }^{1}$ however, PSCC can account for more than $10 \%$ of cancer among men in some parts of Asia, Africa and South America. ${ }^{2}$ Patient prognosis is highly variable, and can be affected by many prognostic factors, especially lymph node involvement status. ${ }^{3}$ Inguinal lymph node dissection (ILND) is the most important method for the diagnosis and treatment for inguinal metastasis, but this technique has a high incidence of complications ${ }^{4}$ and is not necessary for all PSCC patients.

Regular follow-up is important for all patients after complete excision of the penile cancer regardless of whether ILND performed. Accurate prognostication is 
helpful to develop individualized postoperative treatment and follow-up plans. Beyond lymph node status, PSCC prognosis can be also affected by clinicopathological factors such as tumor stage, grade and lymph-vascular invasion (LVI). ${ }^{5,6}$ Models that combine these factors could help accurately predict survival. ${ }^{5,7,8}$ Thuret et al established three prognostic nomograms based on 1324 cases in the Surveillance, Epidemiology, and End Results database (SEER) and separately combined nuclear grade with SEER group/6th TNM stage/6th American Joint Committee on Cancer (AJCC) prognostic stage. ${ }^{7}$ In their internal validation, the predictive accuracies for these three models were $77.8 \%, 80.7 \%$ and $80.9 \%$, respectively. They also created a nomogram based on $\mathrm{T}_{1-3} \mathrm{M}_{0}$ subgroup cases that included variants of 6 th $\mathrm{T}$ stage, $\mathrm{N}$ stage and grade. ${ }^{8}$ This nomogram yielded predictive accuracies of $75.3 \%$ and $78.1 \%$ at 2 and 5 years. In this study, we analyzed the prognostic values of clinicopathological factors in patients from West China Hospital of Sichuan University, evaluated the clinical usefulness of three editions of AJCC TNM stage ${ }^{9-11}$ and the aforementioned four nomograms, ${ }^{7,8}$ and developed a novel nomogram to improve the predictive accuracy of survival in PSCC patients.

\section{Materials and Methods}

\section{Patient Selection}

PSCC patients were selected who had their penile tumor been complete excised by partial or radical penectomy in the West China Hospital at Sichuan University from September 2008 to October 2020, with or without ILND. The exclusion criteria were: a) Eastern Cooperative Oncology Group score higher than one b); unresectable tumor; and c) unwillingness to provide disease-related information. Informed written consent was provided by patients prior to data collection. Finally, 311 PSCC patients were included in this study.

\section{Clinicopathological Features}

Clinical data of patients (diagnostic age, disease duration, smoking history, tumor growth velocity, tumor site, clinical $\mathrm{N}$ stage) were retrieved from the medical records of our institute. Pathological data (tumor size, pathological T stage, grade and LVI) were collected from the pathological reports, which were all provided by Pathological department of our institute. T stage was adjusted according to the AJCC TNM classification system in the 6th, 7th and 8th editions. The clinical $\mathrm{N}$ stage was recorded at 1 month after primary resection (the first time of outpatient visit), and pathological $\mathrm{N}$ stage was recorded if patients underwent ILND.

\section{Follow-Up}

ILND was recommended for patients with a) disease of pT1G2 or higher stage, b) palpable inguinal lymph nodes at 1 month after primary resection, or c) lymph node enlargements found during follow-up. Patients underwent clinical examination every three months in the first year and then every six months. Groin ultrasonic testing was performed every six months for the first two years after operation. The primary outcome was the cancer-specific survival (CSS).

\section{Statistical Methods}

The CSS rate of patients was estimated using the KaplanMeier method, with the prognoses of different subgroups compared by Log rank test. Cox regression analyses were used to identify clinicopathological prognostic factors for PSCC. Factors with statistical significance in the univariate Cox analysis were included in the multivariate analysis, and the prognostic predictive values for these factors were evaluated using the concordance index (c-index). ${ }^{12}$ Patients were scored with three editions of the AJCC prognostic stage group (6th, 7th, and 8th editions) and four prognostic models developed by Thuret et al. ${ }^{7,8}$ The predictive abilities of these outcome models were also tested by c-index. Two novel nomograms were established based on the above prognostic factors and 8th AJCC prognostic stage group. Bootstrapping was used to calculate the corrected c-index, and a calibration curve was created. Receiver operating characteristic (ROC) curves were generated to evaluate the predictive values of the aforementioned factors and nomograms for 2-year CSS. Statistical analyses were performed by MedCalc 19.8 (MedCalc Software Ltd, Ostend, Belgium), The R Programming Language 4.0.4 (R Foundation for Statistical Computing, Vienna, Austria), and SPSS Statistics 25 (IBM Corporation, Armonk, NY, USA), and $\mathrm{P}<0.05$ was considered significant statistically.

\section{Results}

Table 1 shows the clinicopathological data of 311 PSCC patients. The mean age was 54.12 years (standard deviation: 13.75 years), and the median time of follow-up was 
Table I Clinicopathological Features and CSS Rates for 3II PSCC Patients

\begin{tabular}{|c|c|c|c|c|c|}
\hline Variants & Number & I-Year CSS & 2-Year CSS & 5-Year CSS & $P$ value \\
\hline Age of diagnosis (years) & & & & & 0.552 \\
\hline$<50$ & 132 & $88.2 \%$ & $78.9 \%$ & $76.5 \%$ & \\
\hline $50-69$ & 134 & $93.5 \%$ & $85.7 \%$ & $79.2 \%$ & \\
\hline$\geq 70$ & 45 & $90.5 \%$ & $76.2 \%$ & $76.2 \%$ & \\
\hline Smoking history & & & & & 0.827 \\
\hline No & 142 & $90.8 \%$ & $84.8 \%$ & $78.1 \%$ & \\
\hline Yes & 169 & $90.8 \%$ & $79.3 \%$ & $77.2 \%$ & \\
\hline Tumor growth velocity & & & & & 0.100 \\
\hline$<1.5 \mathrm{~cm} /$ recent 3 months & 158 & $91.9 \%$ & $84.1 \%$ & $81.0 \%$ & \\
\hline$\geq 1.5 \mathrm{~cm} /$ recent 3 months & 153 & $88.8 \%$ & $80.2 \%$ & $73.9 \%$ & \\
\hline Tumor location & & & & & 0.078 \\
\hline Prepuse & 15 & $93.3 \%$ & $93.3 \%$ & $93.3 \%$ & \\
\hline Glans of penis & 288 & $90.8 \%$ & $81.8 \%$ & $77.2 \%$ & \\
\hline Body of penis & 8 & $87.5 \%$ & $48.6 \%$ & $48.6 \%$ & \\
\hline Tumor size & & & & & 0.127 \\
\hline$<3 \mathrm{~cm}$ & 149 & $91.3 \%$ & $83.9 \%$ & $81.8 \%$ & \\
\hline$\geq 3 \mathrm{~cm}$ & 162 & $90.3 \%$ & $79.8 \%$ & $73.8 \%$ & \\
\hline ILND operation & & & & & 0.001 \\
\hline No surgery or unknown & 165 & $97.3 \%$ & $94.2 \%$ & $92.1 \%$ & \\
\hline Prophylactic ILND & 28 & $96.3 \%$ & $92.3 \%$ & $87.4 \%$ & \\
\hline Therapeutic ILND & 118 & $80.3 \%$ & $61.8 \%$ & $55.4 \%$ & \\
\hline T stage (8th edition) & & & & & 0.001 \\
\hline $\mathrm{T}_{1}$ & 112 & $95.2 \%$ & $93.0 \%$ & $91.6 \%$ & \\
\hline $\mathrm{T}_{2}$ & 103 & $91.4 \%$ & $77.8 \%$ & $72.7 \%$ & \\
\hline $\mathrm{T}_{3}$ & 88 & $86.2 \%$ & $74.1 \%$ & $68.0 \%$ & \\
\hline $\mathrm{T}_{4}$ & 8 & $71.4 \%$ & $57.1 \%$ & $42.9 \%$ & \\
\hline T stage (7th and 6th editions) ${ }^{\mathrm{a}}$ & & & & & 0.001 \\
\hline$T_{1}$ & 112 & $95.2 \%$ & $93.0 \%$ & $91.6 \%$ & \\
\hline $\mathrm{T}_{2}$ & 174 & $88.4 \%$ & $75.3 \%$ & $71.3 \%$ & \\
\hline $\mathrm{T}_{3}$ & 17 & $94.1 \%$ & $81.6 \%$ & $65.3 \%$ & \\
\hline $\mathrm{T}_{4}$ & 8 & $71.4 \%$ & $57.1 \%$ & $42.9 \%$ & \\
\hline Nuclear grade & & & & & 0.001 \\
\hline Well differentiated & 101 & $99.0 \%$ & $96.6 \%$ & $90.9 \%$ & \\
\hline Moderately differentiated & 148 & $92.7 \%$ & $83.8 \%$ & $79.1 \%$ & \\
\hline Poorly/ undifferentiated & 62 & $72.8 \%$ & $53.0 \%$ & $50.6 \%$ & \\
\hline Lymph vascular invasion & & & & & 0.001 \\
\hline Negative & 287 & $92.3 \%$ & $85.6 \%$ & $82.2 \%$ & \\
\hline Positive & 24 & $74.1 \%$ & $39.5 \%$ & $24.7 \%$ & \\
\hline $\mathrm{N}$ stage (8th edition) & & & & & 0.001 \\
\hline $\mathrm{cN}_{0}$ & $|4|$ & $99.2 \%$ & $97.2 \%$ & $94.7 \%$ & \\
\hline $\mathrm{cN}_{+}$ & 23 & $90.4 \%$ & $80.1 \%$ & $80.1 \%$ & \\
\hline $\mathrm{pN}_{0}$ & 59 & $93.0 \%$ & $89.3 \%$ & $84.9 \%$ & \\
\hline $\mathrm{pN}_{\mathrm{I}}$ & 26 & $95.8 \%$ & $95.8 \%$ & $79.9 \%$ & \\
\hline $\mathrm{pN}_{2}$ & 39 & $79.7 \%$ & $47.5 \%$ & $30.2 \%$ & \\
\hline $\mathrm{pN}_{3}$ & 23 & $42.9 \%$ & $0.0 \%$ & $0.0 \%$ & \\
\hline
\end{tabular}

(Continued) 
Table I (Continued).

\begin{tabular}{|c|c|c|c|c|c|}
\hline Variants & Number & I-Year CSS & 2-Year CSS & 5-Year CSS & $P$ value \\
\hline $\mathrm{N}$ stage (7th edition) & & & & & 0.001 \\
\hline $\mathrm{cN}_{0}$ & $|4|$ & $99.2 \%$ & $97.2 \%$ & $94.7 \%$ & \\
\hline $\mathrm{cN}_{+}$ & 23 & $90.4 \%$ & $80.1 \%$ & $80.1 \%$ & \\
\hline $\mathrm{pN}_{0}$ & 59 & $93.0 \%$ & $89.3 \%$ & $84.9 \%$ & \\
\hline $\mathrm{pN}_{\mathrm{I}}$ & 22 & $94.7 \%$ & $94.7 \%$ & $94.7 \%$ & \\
\hline $\mathrm{pN}_{2}$ & 42 & $81.5 \%$ & $53.4 \%$ & $38.2 \%$ & \\
\hline $\mathrm{pN}_{3}$ & 24 & $43.4 \%$ & $0.0 \%$ & $0.0 \%$ & \\
\hline $\mathrm{N}$ stage (6th edition) & & & & & 0.001 \\
\hline $\mathrm{cN}_{0}$ & $|4|$ & $99.2 \%$ & $97.2 \%$ & $94.7 \%$ & \\
\hline $\mathrm{cN}_{+}$ & 23 & $90.4 \%$ & $80.1 \%$ & $80.1 \%$ & \\
\hline $\mathrm{pN}_{0}$ & 59 & $93.0 \%$ & $89.3 \%$ & $84.9 \%$ & \\
\hline $\mathrm{pN}_{\mathrm{l}}$ & 23 & $94.7 \%$ & $94.7 \%$ & $94.7 \%$ & \\
\hline $\mathrm{pN}_{2}$ & 43 & $78.5 \%$ & $47.7 \%$ & $33.0 \%$ & \\
\hline $\mathrm{pN}_{3}$ & 22 & $53.7 \%$ & $7.4 \% \%$ & $0.0 \%$ & \\
\hline AJCC stage ( 8 and 7 th editions) ${ }^{b}$ & & & & & 0.001 \\
\hline I & 89 & $100.0 \%$ & $98.6 \%$ & $97.0 \%$ & \\
\hline II & 107 & $94.7 \%$ & $91.0 \%$ & $87.1 \%$ & \\
\hline III & 84 & $88.0 \%$ & $73.3 \%$ & $65.7 \%$ & \\
\hline IV & 31 & $53.3 \%$ & $16.4 \%$ & $12.3 \%$ & \\
\hline AJCC stage (6th editions) & & & & & 0.001 \\
\hline 1 & 89 & $100.0 \%$ & $98.6 \%$ & $97.0 \%$ & \\
\hline II & 124 & $94.5 \%$ & $92.3 \%$ & $90.4 \%$ & \\
\hline III & 70 & $84.0 \%$ & $61.2 \%$ & $50.1 \%$ & \\
\hline IV & 28 & $59.5 \%$ & $25.7 \%$ & $20.6 \%$ & \\
\hline Metastasis & & & & & 0.001 \\
\hline No & 274 & $94.7 \%$ & $89.9 \%$ & $88.8 \%$ & \\
\hline Yes & 37 & $64.9 \%$ & $30.4 \%$ & $6.8 \%$ & \\
\hline
\end{tabular}

Notes: ${ }^{a}$ The same classificatory results for 7 th and 6 th $\mathrm{T}$ stage were seen in our cohorts, so they were described together; ${ }^{\mathrm{b}}$ the same classificatory results for 8 th/ 7 th AJCC prognostic stage group were seen in our cohorts, so they were described together.

Abbreviations: CSS, cancer specific survival; PSCC, penile squamous cell carcinoma; Cl, confidence interval; ILND, Inguinal lymph node dissection; AJCC, American Joint Committee on Cancer.

42.34 months (interquartile range: $12.82-81.17$ months). Overall, $46.9 \%$ of patients underwent complete penile surgery and ILND at the beginning of follow-up. During follow-up, 37 patients experienced distant metastasis and 58 patients died of PSCC. The median survival duration for these 58 deceased patients was 12.64 months (interquartile range: 8.83-20.09 months). Survival analyses showed that the two- and five-year CSS rates for all 311 cases and the $\mathrm{pN}_{2-3}$ cohort were $81.8 \% / 77.6 \%$ and $29.0 \%$ / $18.5 \%$, respectively.

In our PSCC cohort, univariate analysis (Table 2) revealed that tumor located in the penis body, higher $\mathrm{T}$ and $\mathrm{N}$ stages (6th, 7th and 8th editions), poorer nuclear grade, and LVI were significantly correlated with poorer survival $(\mathrm{P}<0.05)$. Significant poorer survival was also seen in higher
AJCC prognostic stage group (6th, 7th and 8th editions). The results showed that smoking, large tumor diameter, and rapid tumor growth were potential prognostic risk factors (Hazard ratio $>1$ ), but there were no significant differences (all $\mathrm{P}>0.05$ ). It is interesting that a tendency toward better prognosis was found in patients between 50 and 70 years old, compared with younger and older subgroups.

Two different Cox models were established in multivariate analysis (Supplementary Table S1). The first Cox model combined tumor location, nuclear grade and LVI with 8th T and $\mathrm{N}$ stage separately, and the multivariate analysis showed that only $\mathrm{N}$ stage was an independent predictor for survival $(\mathrm{P}<0.05)$. The second Cox model replaced $\mathrm{T}$ and $\mathrm{N}$ stage as the 8th AJCC prognostic stage group, and only AJCC stages were independently correlated with survival $(\mathrm{P}<0.05)$. 
Table 2 Univariate Analysis for CSS in 3II PSCC Patients

\begin{tabular}{|c|c|c|c|}
\hline Clinical Pathological Data & $\mathbf{P}$ value & Hazard Ratio & $95 \% \mathrm{Cl}$ \\
\hline Smoking $^{\mathrm{a}}$ & 0.827 & 1.059 & $0.631-1.778$ \\
\hline $50 \leq$ Diagnosis age $^{2} 70$ years $^{\mathrm{b}}$ & 0.476 & 0.752 & $0.344-1.644$ \\
\hline Diagnosis age $>70$ years $^{b}$ & 0.967 & 1.016 & $0.479-2.155$ \\
\hline Tumor in glans penis ${ }^{c}$ & 0.194 & 3.706 & $0.512-26.809$ \\
\hline Tumor in body of penis ${ }^{c}$ & 0.048 & 9.830 & $1.019-94.802$ \\
\hline Tumor size $\geq 3 \mathrm{~cm}^{\mathrm{d}}$ & 0.130 & $\mathrm{I} .50 \mathrm{I}$ & $0.887-2.541$ \\
\hline Tumor growth $\geq 1.5 \mathrm{~cm} /$ recent 3 months ${ }^{\mathrm{e}}$ & 0.103 & 1.542 & $0.917-2.593$ \\
\hline$T_{2}$ (7th and 6th editions) ${ }^{f}$ & 0.001 & 3.919 & $1.836-8.367$ \\
\hline$T_{3}$ (7th and 6th editions) ${ }^{f}$ & 0.015 & 4.007 & $1.310-12.255$ \\
\hline$T_{4}$ (7th and 6th editions) ${ }^{f}$ & 0.001 & 9.643 & $2.896-32.112$ \\
\hline$T_{2}$ (8th edition) ${ }^{f}$ & 0.003 & 3.360 & $1.495-7.553$ \\
\hline$T_{3}$ (8th edition) $^{f}$ & 0.001 & 4.652 & $2.087-10.366$ \\
\hline$T_{4}$ (8th edition) ${ }^{f}$ & 0.001 & 9.647 & $2.897-32.123$ \\
\hline Moderately differentiated ${ }^{g}$ & 0.011 & 2.968 & $1.286-6.848$ \\
\hline Poorly/undifferentiated ${ }^{g}$ & 0.001 & 9.492 & $4.067-22.157$ \\
\hline Lymph vascular invasion ${ }^{h}$ & 0.001 & 5.990 & $3.285-10.924$ \\
\hline $\mathrm{cN}+(8 \text { th edition })^{\mathrm{i}}$ & 0.056 & 2.983 & $0.973-9.152$ \\
\hline $\mathrm{pN}_{\mathrm{I}}(8 \text { th edition })^{\mathrm{i}}$ & 0.363 & 1.790 & $0.510-6.285$ \\
\hline $\mathrm{pN}_{2}(8 \text { th edition })^{\mathrm{i}}$ & 0.001 & 14.822 & $7.243-30.332$ \\
\hline $\mathrm{pN}_{3}(8 \text { th edition })^{\mathrm{i}}$ & 0.001 & 58.254 & $26.594-127.605$ \\
\hline $\mathrm{cN}+(7 \text { th edition })^{\mathrm{i}}$ & 0.056 & 2.986 & $0.974-9.160$ \\
\hline $\mathrm{pN}_{\mathrm{I}}(7 \text { th edition })^{\mathrm{i}}$ & 0.169 & 2.417 & $0.688-8.489$ \\
\hline $\mathrm{pN}_{2}(7 \text { th edition })^{\mathrm{i}}$ & 0.001 & $10.88 \mid$ & $5.344-22.155$ \\
\hline $\mathrm{pN}_{3}(7 \text { th edition })^{\mathrm{i}}$ & 0.001 & 55.582 & $25.528-121.020$ \\
\hline $\mathrm{cN}+(6 \text { th edition })^{\mathrm{i}}$ & 0.013 & 3.708 & $1.322-10.403$ \\
\hline $\mathrm{pN}_{\mathrm{I}}\left(\right.$ (6th edition) ${ }^{\mathrm{i}}$ & 0.173 & 2.393 & $0.68 I-8.404$ \\
\hline $\mathrm{pN}_{2}$ (6th edition) ${ }^{\mathrm{i}}$ & 0.001 & 12.208 & $6.082-24.505$ \\
\hline $\mathrm{pN}_{3}$ (6th edition) ${ }^{\mathrm{i}}$ & 0.001 & 34.963 & $|6.17|-75.596$ \\
\hline AJCC group II (8th and 7th editions) ${ }^{\mathrm{i}}$ & 0.039 & 4.966 & $1.088-22.673$ \\
\hline AJCC group III (8th and 7th editions) ${ }^{\mathrm{j}}$ & 0.001 & 17.693 & $4.177-74.947$ \\
\hline AJCC group IV (8th and 7 th editions) ${ }^{\mathrm{i}}$ & 0.001 & 92.914 & $21.591-399.845$ \\
\hline AJCC group II (6th edition) ${ }^{j}$ & 0.043 & 4.752 & $1.053-21.444$ \\
\hline AJCC group III (6th edition) ${ }^{\mathrm{j}}$ & 0.001 & 25.505 & $6.065-107.408$ \\
\hline AJCC group IV (6th edition) ${ }^{\mathrm{j}}$ & 0.001 & 69.474 & $15.975-302.144$ \\
\hline
\end{tabular}

Notes: ${ }^{a}$ Reference group is no-smoker; ${ }^{b}$ reference group is diagnosis age $<50$; ${ }^{c}$ reference group is tumor in prepuce; ${ }^{d}$ reference group is tumor size $<3 \mathrm{~cm}$; ${ }^{\mathrm{e}}$ reference group is Tumor growth $<0.5 \mathrm{~cm} /$ month; the same classificatory results for 7 th and 6 th $\mathrm{T}$ stage were seen in our cohorts, so they were described together. The reference group is $\mathrm{T}_{1 ;}{ }^{g}$ reference group is nuclear well differentiated group; ${ }^{\mathrm{h}}$ reference group is lymph vascular invasion negative group; 'reference group is c $\mathrm{N}_{0} / \mathrm{p} \mathrm{N}_{0}$ group; ${ }^{\mathrm{i}}$ the same classificatory results for $8 \mathrm{th} / 7$ th AJCC prognostic stage group were seen in our cohorts, so they were described together. The reference group is AJCC group I.

Abbreviations: CSS, cancer specific survival; PSCC, penile squamous cell carcinoma; Cl, confidence interval; AJCC, American Joint Committee on Cancer.

The predictive abilities of the above prognostic factors were evaluated (Table 3). $\mathrm{N}$ stage showed the strongest predictive accuracy (c-indexes: $0.814,0.811$ and 0.801 for 8th, 7th, and 6th editions), followed by nuclear grade (c-index: 0.711). However, none of the other factors had predictive accuracies above 0.70. External evaluations for AJCC prognostic stage group and four previously established prognostic nomograms were also performed (Table 3). Among them, the nomogram using the 6th AJCC edition and nuclear grade had the highest predictive accuracy (c-index: 0.837).
To better predict PSCC prognosis, two novel nomograms were created (Figures 1 and 2; calibration curves shown in Supplementary Figures 1 and 2). In novel nomogram 1, we included 8th T stage, $\mathrm{N}$ stage, tumor location, nuclear grade, and LVI. The bias-corrected c-index of the model was 0.870 (95\% confidence interval: 0.829-0.911), which was better than those of AJCC prognostic stage group and four previous models. Novel nomogram 2 included tumor location, nuclear grade and LVI, but $\mathrm{T}$ and $\mathrm{N}$ stage were replaced by AJCC stage. The bias-corrected c-index of the model was 0.855 ( $95 \%$ confidence interval: $0.815-0.896$ ). 
Table 3 Predictive Value of Different Variants and Prognostic Models in 311 PSCC Patients

\begin{tabular}{|c|c|c|}
\hline Variants and Prognostic Models & c-Index & $(95 \% \mathrm{Cl})$ \\
\hline Tumor site $^{\mathrm{a}}$ & 0.535 & $0.496-0.573$ \\
\hline T stage (8th edition) & 0.650 & $0.582-0.718$ \\
\hline $\mathrm{T}$ stage $(7 \text { th and } 6 \text { th edition })^{\mathrm{b}}$ & 0.630 & $0.569-0.691$ \\
\hline $\mathrm{N}$ stage (8th edition) ${ }^{c}$ & 0.814 & $0.756-0.873$ \\
\hline $\mathrm{N}$ stage (7th edition) ${ }^{c}$ & 0.811 & $0.752-0.869$ \\
\hline $\mathrm{N}$ stage (6th edition) ${ }^{c}$ & 0.801 & $0.744-0.859$ \\
\hline Nuclear grade & 0.711 & $0.65 I-0.77 \mid$ \\
\hline Lymph vascular invasion & 0.600 & $0.548-0.653$ \\
\hline AJCC stage ( 8 and 7 th editions) ${ }^{d}$ & 0.821 & $0.774-0.868$ \\
\hline AJCC stage (6th edition) & 0.823 & $0.777-0.869$ \\
\hline SEER + grade nomogram ${ }^{e}$ & 0.738 & $0.68 \mathrm{I}-0.795$ \\
\hline TNM + grade nomogram ${ }^{e}$ & 0.792 & $0.739-0.846$ \\
\hline $\mathrm{AJCC}+$ grade nomogram ${ }^{\mathrm{e}}$ & 0.831 & $0.787-0.875$ \\
\hline $\mathrm{T}_{1-3}+\mathrm{N}+$ grade nomogram ${ }^{f}$ & 0.781 & $0.710-0.851$ \\
\hline Novel nomogram I & 0.870 & $0.829-0.911$ \\
\hline Novel nomogram 2 & 0.855 & $0.815-0.896$ \\
\hline
\end{tabular}

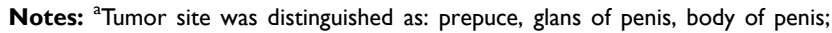
${ }^{b}$ the same classificatory results for 7th and 6th T stage were seen in our cohorts, so they were described together; 'lymph node status was distinguished as: $\mathrm{cN}_{0} / \mathrm{pN}_{0}$, $\mathrm{cN}_{+}, \mathrm{pN}_{1}, \mathrm{pN}_{2}, \mathrm{pN}_{3} .{ }^{\mathrm{d}}$ The same classificatory results for 8th/ 7th AJCC prognostic stage group were seen in our cohorts, so they were described together; ${ }^{\mathrm{e}}$ reported by Thuret et al; ${ }^{7}$ reported by Thuret et al. ${ }^{8}$

Abbreviations: PSCC, penile squamous cell carcinoma; $\mathrm{Cl}$, confidence interval; SEER, Surveillance, Epidemiology, and End Results program; AJCC, American Joint Committee on Cancer.

As our results showed that the majority of cancerspecific death occurred within 2 years after surgery, the predictive effects of previously reported models and novel nomograms for 2-year CSS were evaluated with ROC curves. Figure 3A shows the predictive accuracies of models for 2-year survival in all 311 PSCC cases. The top two accuracies were for novel nomograms 1 and 2, with AUCs of 0.893 and 0.873 , respectively. The prediction efficacy of the SEER stage + grade nomogram was the poorest performing models $(\mathrm{AUC}=0.752)$. This analysis was also performed in the $\mathrm{T}_{1-3}$ subgroup (303 patients), so the 6th $\mathrm{T}_{1-3}+\mathrm{N}+$ grade model nomogram could be also evaluated ${ }^{8}$ (Figure 3B). In this subgroup analysis, the ranking of prediction accuracies of different models was similar with a previous analysis in all 311 cases, and the 6th $\mathrm{T}_{1-3}+\mathrm{N}+$ grade model nomogram was second to the last $(\mathrm{AUC}=0.802)$.

\section{Discussion}

This study analyzed the correlation between clinicopathological factors and CSS of postoperative PSCC patients. We externally validated the predictive effects of the 6th to 8th versions of AJCC prognostic stage and four prognostic nomograms reported previously, then constructed two novel nomograms and compared their predictive ability with previous stage groups and nomograms. We developed new prediction scoring models using the 8th TN stage/ AJCC stage group with tumor location, nuclear grade and LVI in a large sample size with long-term follow-up.

Lymphatic status is the most important prognostic factor for PSCC. ${ }^{13}$ Patients with no evidence of inguinal metastasis have an average 5-year survival rate of $87 \%$ to $100 \% .{ }^{14-16}$ The prognosis for patients with lymphatic metastasis is significantly poorer, with an average 5 -year survival of $\sim 60 \%{ }^{13}$ This metastatic population can be further classified using the different version of AJCC $\mathrm{N}$ stage. ${ }^{9-11}$ Significantly better 5-year survival rates were observed in patients with no more than two unilateral metastatic nodes compared to those with more positive nodes (73-88\% vs 7-50\%). ${ }^{16-19}$ After further subdivision, superior survival was still observed in patients with one positive node compared to two unilateral positive nodes, ${ }^{20}$ and both cases can be considered as minimal nodal disease. ${ }^{13}$ For those patients with extranodal-extension or pelvic nodes metastasis, the 5-year survival rate can decrease to $<14 \% .{ }^{13,16}$ The 8 th AJCC $\mathrm{N}$ stage was designed based on these data, which was slightly different from former editions. ${ }^{9-11}$ Our study showed similar survival rates in different lymph node status as previous studies, with better predictive accuracy of survival for 8 th $\mathrm{N}$ stage compared with the 6th and 7th editions. Hence, the latest $\mathrm{N}$ stage version was used in our novel model.

Although clinical $\mathrm{N}$ stage was demonstrated as one of the most important predictors for lymphatic metastasis in several studies, ${ }^{21-23}$ it cannot reflect the true pathological status of PSCC. One analysis revealed a false-positive rate that ranged from $8 \%$ to $65 \%$ and a false-negative rate that ranged from $2 \%$ to $100 \% .{ }^{13}$ Infection in primary tumor, obesity, and low specificity of traditional imaging methods might have contributed these results. Therefore, in our study, patients who did not undergo ILND were differentiated according to the presence or absence of clinically positive lymph nodes; no further subdivision was conducted on this basis.

Primer tumor invasion status, LVI and nuclear grade, also significantly influence PSCC prognosis. ${ }^{5-8,24}$ Tumor invasion status in corpus cavernosa, corpus spongiosum and urethra is significantly correlated with lymphatic metastasis and poor prognosis in several studies. ${ }^{5,6,22,23}$ However, a lower rate of lymphatic metastasis and better survival were seen in cohorts with invasion into the 
Points

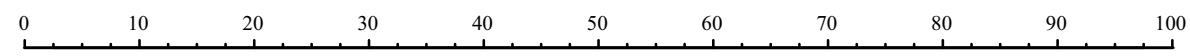

Tumor site

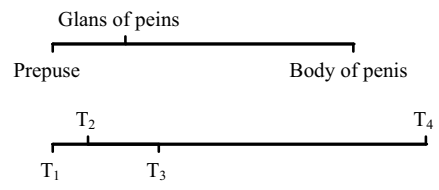

$8^{\text {th }} \mathrm{T}$ stage

$8^{\text {th }} \mathrm{N}$ stage

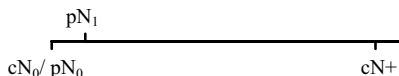

$\mathrm{pN}_{2}$

$\mathrm{cN}_{0} / \mathrm{pN}_{0}$

Intermediate differentiated

Nuclear grade

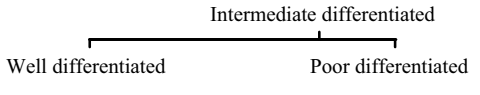

Lymph vascular invasion

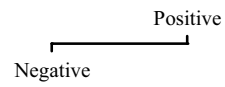

Total Points

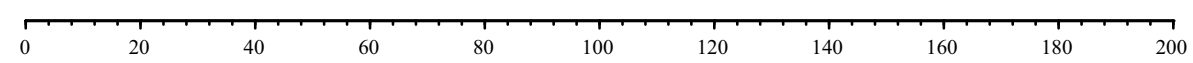

1 year cancer specific survival probability

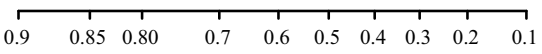

2 years cancer specific survival probability

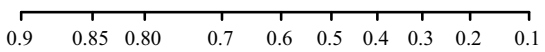

5 years cancer specific survival probability

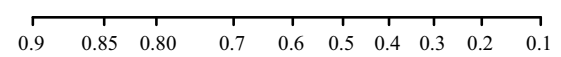

Figure I Novel nomogram I predicting cancer-specific survival of postoperative PSCC patients.

Points

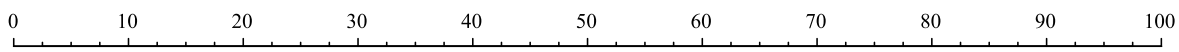

Tumor site

$\stackrel{\text { Glans of peins }}{\text { Body of penis }}$

$8^{\text {th }}$ AJCC prognostic stage

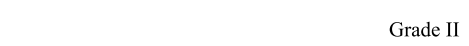

Grade IV

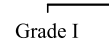

Grade II

-

Nuclear grade

Intermediate differentiated

Well differentiated Poor differentiated

Lymph vascular invasion

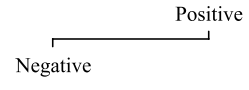

Total Points

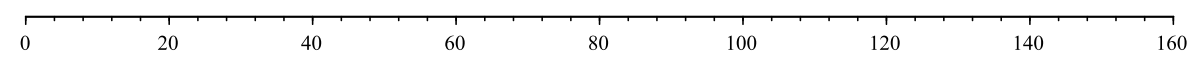

1 year cancer specific survival probability

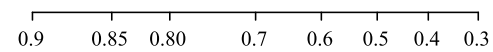

2 years cancer specific survival probability

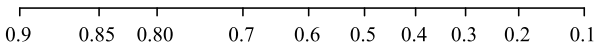

5 years cancer specific survival probability

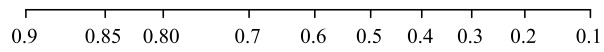

Figure 2 Novel nomogram 2 predicting cancer-specific survival of postoperative PSCC patients.

Abbreviation: AJCC, American Joint Committee on Cancer. 

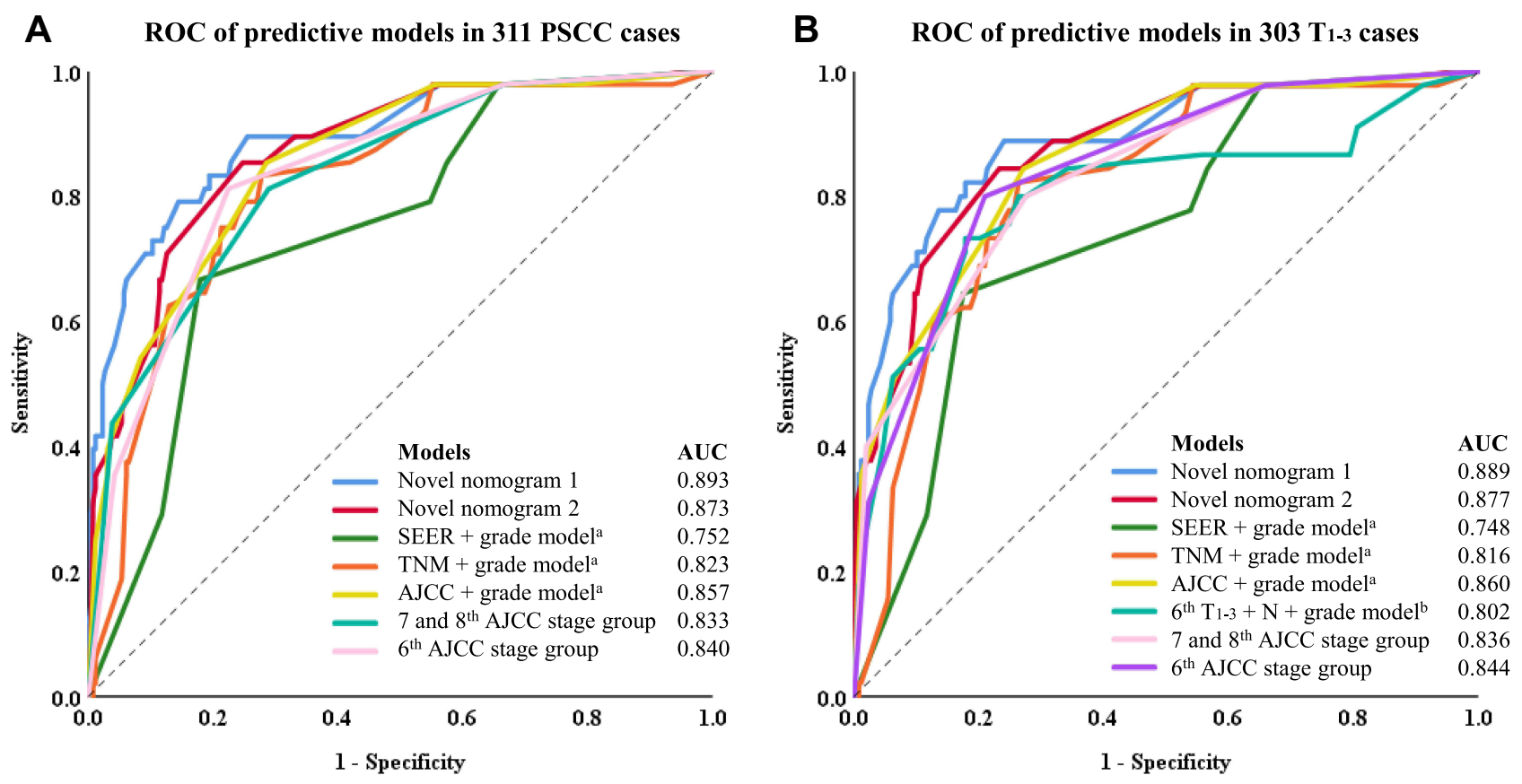

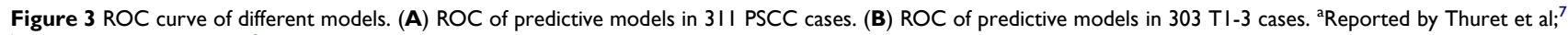
breported by Thuret et al. ${ }^{8}$

Abbreviations: AJCC, American Joint Committee on Cancer; AUC, Area Under the Curve; ROC, Receiver Operating Characteristic Curve; SEER, Surveillance, Epidemiology, and End Results Program.

corpus spongiosum versus corpora cavernosa. ${ }^{25,26}$ The most recent 8th $\mathrm{T}$ stage revised the definition of $\mathrm{pT}_{2}$ disease to tumor invading into the corpus spongiosum and $\mathrm{pT}_{3}$ for corpora cavernosum invasion according to results of papers aforementioned, and we also demonstrated better prognostication for the latest $\mathrm{T}$ stage than former editions. Besides, although the AJCC 7th and 8th $\mathrm{T}$ stage separate T1a and T1b patients based on LVI status, we propose that LVI should be independently used as prognostic predictor. Our cohort did not include any T1b patients and patients with, and patients with LVI had poorer survival than those without regardless of tumor site, stage, nuclear grade and so other factors.

Tumor location was included in our model, and was significant correlated with prognosis. In a recent study by Reyes et al, the risk of death for patients with disease in the prepuce/penis corpus/overlapping lesion was two-fold than that of patients with disease in gland. ${ }^{27}$ However, this difference was not significant in a recent study based on populations from the SEER database. ${ }^{28}$ Anatomically, tumors located in the body of penis seem to be closer to the cavernosum, where it is easier to penetrate the thin skin of the penis and invade the cavernosum. The prognostic value of tumor site still needs further verification.
Although no significant prognostic difference was found between age subgroups, there was an interesting trend that patients between 50 and 70 years old had better survival than younger or older patients. In a series of 378 patients reported by Paiva et al, the highest rate of cancerspecific death was in patients $<40$ years old compared with older cases (19\% vs $11-13 \%)$, and these younger patients were more likely to experience tumor recurrence and growth. ${ }^{29}$ However, two other groups identified young age as protective factors for survival. ${ }^{27,28}$ Considering these nonsignificant and inconsistent results, we do not think age is a useful predictive factor for PSCC survival. In terms of tumor size and growth velocity, there were trends that large and fast-growing tumors might be related to poor prognosis. However, we did not find significant cut-off values for these variables. Future studies with larger sample sizes and longer follow-up time will hopefully clarify this issue.

To further predict PSCC survival, several prognostic models combining the aforementioned factors were established. Our study demonstrated that the predictive effect of 8th AJCC prognostic stage group did not improve with the addition of $\mathrm{T}$ and $\mathrm{N}$ stages, suggesting that it is more rational to consider these independently in prognostic 
models. This was also confirmed by our comparison of nomograms 1 and 2. The lower predictive accuracies in our external validation of four previous nomograms also indicate that former edition of TNM stage might not be that suitable for prognostic models. In fact, similar external validation results were also reported by Zhu et al for three of the aforementioned nomograms, with c-indexes of $0.728,0.817$, and 0.832 for SEER + grade nomogram, 6th $\mathrm{TNM}+$ grade nomogram, and 6th AJCC + grade nomogram, respectively. ${ }^{24}$ Our novel predictive model included the latest versions of $\mathrm{T}$ and $\mathrm{N}$ stages in combination with other important predictive factors and showed better predictive accuracy than previous published models. It is anticipated that this novel model may have greater application value for clinical practice.

Our results should be considered in the context of some limitations. For one thing, the population used for nomogram establishing came from a single source, which means usefulness of our new model in populations of other racial background or geographic regions could not be evaluated for the moment. However, the good thing is that systematic errors such as differences of medical-care conditions between populations of different religion and race can be reduced by a homogeneous model-created population. For another, molecular targets that may be associated with survival were not analyzed in this study. Since the present nomogram incorporated the most important and routine clinicopathological factors, we hope that this approach will facilitate clinical decision making in a more generalizable way.

\section{Conclusions}

Tumor site, $\mathrm{T}$ stage, $\mathrm{N}$ stage, nuclear grade, and LVI are important predictors of survival in patients with PSCC. The 8th $\mathrm{T}$ and $\mathrm{N}$ stages have better predictive value for survival than 6th and 7th edition. In external validation of three versions of AJCC prognostic stage group and four nomograms, each edition of AJCC stage groups and two nomograms (6th AJCC stage group plus grade, 6th TNM stage plus group) had c-indexes $>0.800$. Two novel nomograms were developed that included prognostic predictors, and both showed better predictive accuracy than the above prognostic models. Nomogram 1 (8th $\mathrm{T}$ and $\mathrm{N}$ stage, tumor site, grade, LVI) had a better predictive effect than nomogram 2 (8th AJCC prognostic stage group, tumor site, grade, LVI).

\section{Data Sharing Statement}

The data that support the findings of this study are available on request from the corresponding author.

\section{Ethics Approval and Consent to Participate}

This retrospective study was approved Ethics Committee of West China Hospital, Sichuan University, with the whole process supervised. Patients and their authorized family members had been fully informed before follow-up work was performed, with informed consent signed. This study was conducted in accordance with the Declaration of Helsinki.

\section{Consent for Publication}

Consent for publication was obtained from all participants.

\section{Acknowledgments}

We would like to thank the Surveillance, Epidemiology, and End Results database in providing high quality clinical data for our research.

\section{Author Contributions}

All authors made substantial contributions to conception and design, acquisition of data, or analysis and interpretation of data; took part in drafting the article or revising it critically for important intellectual content; agreed to submit to the current journal; gave final approval of the version to be published; and agree to be accountable for all aspects of the work.

\section{Funding}

This study was supported by the Science and Technology Foundation of Sichuan Province (2017JY0226), the China Scholarship Council (Reference number: 202006240333) and the 1.3.5 Project for Disciplines of Excellence, West China Hospital, Sichuan University (Reference number: ZY2016104).

\section{Disclosure}

The authors report no conflicts of interest in this work.

\section{References}

1. Backes DM, Kurman RJ, Pimenta JM, Smith JS. Systematic review of human papillomavirus prevalence in invasive penile cancer. Cancer Causes Control. 2009;20(4):449-457. doi:10.1007/s10552-008-9276-9

2. Alemany L, Cubilla A, Halec G, et al. Role of human papillomavirus in penile carcinomas worldwide. Eur Urol. 2016;69(5):953-961.

3. Leone A, Diorio GJ, Pettaway C, Master V, Spiess PE. Contemporary management of patients with penile cancer and lymph node metastasis. Nat Rev Urol. 2017;14(6):335-347.

4. Koifman L, Hampl D, Koifman N, Vides AJ, Ornellas AA. Radical open inguinal lymphadenectomy for penile carcinoma: surgical technique, early complications and late outcomes. J Urol. 2013;190 (6):2086-2092. 
5. Kattan MW, Ficarra V, Artibani W, et al. Nomogram predictive of cancer specific survival in patients undergoing partial or total amputation for squamous cell carcinoma of the penis. $J$ Urol. 2006;175 (6):2103-8; discussion 8 .

6. Novara G, Galfano A, De Marco V, Artibani W, Ficarra V. Prognostic factors in squamous cell carcinoma of the penis. Nat Clin Pract Urol. 2007;4(3):140-146.

7. Thuret R, Sun M, Abdollah F, et al. Tumor grade improves the prognostic ability of American Joint Committee on Cancer stage in patients with penile carcinoma. J Urol. 2011;185(2):501-507.

8. Thuret R, Sun M, Abdollah F, et al. Conditional survival predictions after surgery for patients with penile carcinoma. Cancer. 2011;117 (16):3723-3730.

9. Amin MB, Edge S, Greene F, et al. AJCC Cancer Staging Manual Eighth Edition. Springer; 2017.

10. Edge SB, Byrd DR, Compton CC, Fritz AG, Greene FL, Trotti A. AJCC Cancer Staging Manual Seventh Edition. Spinger; 2010.

11. Frederick LG, Page DL, Fleming ID, et al. AJCC Cancer Staging Manual Sixth Edition. Spinger; 2003.

12. Harrell FE Jr, Lee KL, Mark DB. Multivariable prognostic models: issues in developing models, evaluating assumptions and adequacy, and measuring and reducing errors. Stat Med. 1996;15(4):361-387. doi:10.1002/(SICI)1097-0258(19960229)15:4<361::AID-SIM168>3. $0 . \mathrm{CO} ; 2-4$

13. Pettaway CA, Crook JM, Pagliaro LC; FRCPC. Tumors of the penis. In: Wein AJ, editor. Campbell-Walsh Urology. 11th ed. Philadelphia, USA: Elsevier; 2016: 846-878.

14. Horenblas S, van Tinteren H, Delemarre JF, Moonen LM, Lustig V, van Waardenburg EW. Squamous cell carcinoma of the penis. III. Treatment of regional lymph nodes. J Urol. 1993;149(3):492-497.

15. Ornellas AA, Seixas AL, Marota A, Wisnescky A, Campos F, de Moraes JR. Surgical treatment of invasive squamous cell carcinoma of the penis: retrospective analysis of 350 cases. J Urol. 1994;151 (5):1244-1249.

16. Ravi R. Prophylactic lymphadenectomy vs observation vs inguinal biopsy in node-negative patients with invasive carcinoma of the penis. Jpn J Clin Oncol. 1993;23(1):53-58.

17. Fraley EE, Zhang G, Manivel C, Niehans GA. The role of ilioinguinal lymphadenectomy and significance of histological differentiation in treatment of carcinoma of the penis. J Urol. 1989;142 (6):1478-1482.

18. Pandey D, Mahajan V, Kannan RR. Prognostic factors in node-positive carcinoma of the penis. J Surg Oncol. 2006;93 (2):133-138
19. Graafland NM, van Boven HH, van Werkhoven E, Moonen LM, Horenblas S. Prognostic significance of extranodal extension in patients with pathological node positive penile carcinoma. $J$ Urol. 2010;184(4):1347-1353.

20. Li ZS, Yao K, Chen $\mathrm{P}$, et al. Modification of $\mathrm{N}$ staging systems for penile cancer: a more precise prediction of prognosis. $\mathrm{Br} J$ Cancer. 2015;112(11):1766-1771.

21. Peak TC, Russell GB, Dutta R, Rothberg MB, Chapple AG, Hemal AK. A national cancer database-based nomogram to predict lymph node metastasis in penile cancer. BJU Int. 2019;123 (6):1005-1010.

22. Zhu Y, Zhang HL, Yao XD, et al. Development and evaluation of a nomogram to predict inguinal lymph node metastasis in patients with penile cancer and clinically negative lymph nodes. $J$ Urol. 2010;184(2):539-545.

23. Ficarra V, Zattoni F, Artibani W, et al. Nomogram predictive of pathological inguinal lymph node involvement in patients with squamous cell carcinoma of the penis. J Urol. 2006;175(5):1700-4;discussion 4-5.

24. Zhu Y, Gu WJ, Ye DW, et al. External validation of nomograms for predicting cancer-specific mortality in penile cancer patients treated with definitive surgery. Chin J Cancer. 2014;33(5):249-255.

25. Sun M, Djajadiningrat RS, Alnajjar HM, et al. Development and external validation of a prognostic tool for prediction of cancer-specific mortality after complete loco-regional pathological staging for squamous cell carcinoma of the penis. BJU Int. 2015;116(5):734-743.

26. Leijte JA, Gallee M, Antonini N, Horenblas S. Evaluation of current TNM classification of penile carcinoma. J Urol. 2008;180(3):933-8; discussion 8.

27. Reyes ME, Borges H, Adjao MS, Vijayakumar N, Spiess PE, Schabath MB. Novel prognostic models for patients with penile carcinoma. Cancer Control. 2020;27(1):1073274820924728.

28. Yang J, Pan Z, He Y, et al. Competing-risks model for predicting the prognosis of penile cancer based on the SEER database. Cancer Med. 2019;8(18):7881-7889.

29. Paiva GR, de Oliveira Araujo IB, Athanazio DA, de Freitas LA. Penile cancer: impact of age at diagnosis on morphology and prognosis. Int Urol Nephrol. 2015;47(2):295-299.

\section{Publish your work in this journal}

Cancer Management and Research is an international, peer-reviewed open access journal focusing on cancer research and the optimal use of preventative and integrated treatment interventions to achieve improved outcomes, enhanced survival and quality of life for the cancer patient.
The manuscript management system is completely online and includes a very quick and fair peer-review system, which is all easy to use. Visit http://www.dovepress.com/testimonials.php to read real quotes from published authors. 